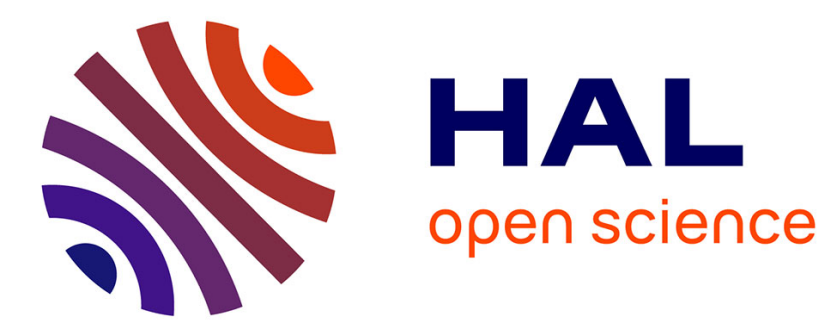

\title{
Développement in vitro d'embryons immatures de Lupinus albus L. et de Lupinus mutabilis Sweet par culture de gousses, d'ovules ou d'embryons isolés
}

Elisabeth Vuillaume, Tine Hoff

\section{- To cite this version:}

Elisabeth Vuillaume, Tine Hoff. Développement in vitro d'embryons immatures de Lupinus albus L. et de Lupinus mutabilis Sweet par culture de gousses, d'ovules ou d'embryons isolés. Agronomie, 1986, 6 (10), pp.925-930. hal-00884841

\section{HAL Id: hal-00884841 https://hal.science/hal-00884841}

Submitted on 1 Jan 1986

HAL is a multi-disciplinary open access archive for the deposit and dissemination of scientific research documents, whether they are published or not. The documents may come from teaching and research institutions in France or abroad, or from public or private research centers.
L'archive ouverte pluridisciplinaire HAL, est destinée au dépôt et à la diffusion de documents scientifiques de niveau recherche, publiés ou non, émanant des établissements d'enseignement et de recherche français ou étrangers, des laboratoires publics ou privés. 


\section{Développement in vitro d'embryons immatures de Lupinus albus L. et de Lupinus mutabilis Sweet par culture de gousses, d'ovules ou d'embryons isolés}

Elisabeth VUILLAUME \& Tine HOFF

I.N.R.A., Station d'Amélioration des Plantes, Centre de Recherches de Dijon, BV 1540, F 21034 Dijon Cedex

\begin{abstract}
Pour les espèces Lupinus mutabilis Sweet et Lupinus albus L. nous avons pu obtenir la germination d'embryons de $1 \mathrm{~mm}$ et plus, en culture in vitro sur milieu solide ne contenant pas d'hormones.

Pour les embryons plus petits nous avons essayé de cultiver des gousses, des ovules ou des moitiés d'ovules contenant albumen et embryon, sur milieu solide ou en milieu liquide. La culture de gousses en milieu liquide et la culture d'ovules sur milieu solide ne permettent pas le sauvetage des embryons de $L$. albus, $L$. mutabilis n'ayant pas été étudié pour ces types de culture. Le développement des ovules et des embryons est possible, soit en cultivant des ovules sur papier filtre formant un pont au-dessus du milieu liquide, soit par la culture de demi-ovules noyés dans un milieu à faible teneur en agar $(5 \mathrm{~g} / \mathrm{l})$.
\end{abstract}

Mots clés additionnels : Sauvetage d'embryon.
In vitro development of immature embryos of Lupinus albus $L$. and Lupinus mutabilis Sweet by culture of pods, ovules or isolated embryos.

In Lupinus mutabilis Sweet and Lupinus albus L., embryos of $1 \mathrm{~mm}$ or more germinated in vitro on solid media without hormones to grow. An attempt was made for smaller embryos of $L$. albus by culturing pods, ovules or half ovules containing endosperm and embryo, in liquid or on solid media. Pod culture in liquid medium and ovule culture on solid medium did not allow embryo development. However, embryos could be grown either by culturing ovules on a paper bridge over liquid medium or by embedding half ovules in a medium with a lowered agar concentration $(5 \mathrm{~g} / \mathrm{l})$.

Additional key words : Embryo rescue.

\section{INTRODUCTION}

L'hybridation entre Lupinus albus L. et Lupinus mutabilis Sweet, en rendant possible la recombinaison des caractères des 2 espèces, devrait permettre de résoudre quelques-uns des problèmes rencontrés par la sélection de ces espèces (Vuillaume \& HOFF, 1986).

Des hybridations entre les espèces européennes cultivées ( $L$. albus, L. angustifolius $\mathrm{L}$. et L. luteus L.) ont été réalisés par JARANOVSKI (1962). L'étude histologique des gousses obtenues par JARANOVSKI a apporté les résultats suivants :

1) la formation d'une gousse après un croisement est le signe que la fécondation a bien eu lieu ;

2) le développement des embryons ne dépasse jamais le stade cordiforme, l'arrêt pouvant être beaucoup plus précoce (stade $1^{\text {re }}$ division par exemple) ;

3) l'arrêt au stade cordiforme semble être dû au développement aberrant soit du suspenseur soit de l'albumen.

Des hybridations entre espèces européennes et $L$. mutabilis ont été faites par WiLliams et al. (1980). Ceux-ci ont pu observer que :

1) le stigmate des fleurs de L. mutabilis ne permet pas la germination du pollen des espèces européennes alors que son pollen peut germer dans les styles de ces espèces. (Le sens du croisement ne semble pas avoir d'effet sur les pourcentages de fécondation entre espèces européennes) ; 
2) il y a formation de gousses contenant des ovules (fécondés ?) de taille réduite.

$\mathrm{Au}$ vu de ces travaux, il apparaît que la barrière d'incompatibilité dans ces hybridations interspécifiques est généralement postzygotique. Le sauvetage des embryons immatures peut donc être le moyen de lever ces incompatibilités.

Dans la mesure où les embryons interspécifiques sont difficiles à obtenir (10 à 25 p. 100 de nouaison dans les meilleurs des cas), nous avons décidé de mettre au point des techniques de culture in vitro assurant le développement embryonnaire jusqu'à son terme pour $L$. albus et de $L$. mutabilis. Ces techniques ont été qualifiées par les Anglo-Saxons de «sauvetage d'embryon "s.

Davies \& Williams (comm. pers.) ont étudié le développement et la croissance des embryons de diverses légumineuses dont $L$. albus et $L$. mutabilis. Ils ont notamment établi que le stade cordiforme est atteint, chez $L$. albus, entre le $24^{\mathrm{e}}$ et le $28^{\mathrm{e}}$ j après la date du croisement. L'embryon mesure alors de 300 à $800 \mu \mathrm{m}$. Pour $L$. mutabilis ce stade est atteint entre le $12^{\mathrm{e}}$ et le $16^{\mathrm{e}} \mathrm{j}$ après croisement, l'embryon mesurant entre 150 et $500 \mu \mathrm{m}$. Compte tenu de ces résultats, nous avons donc tenté d'obtenir la poursuite du développement des embryons de moins de $500 \mu \mathrm{m}$ et la germination d'embryons au stade torpedo qui mesurent environ $1 \mathrm{~mm}$.

Pour résoudre les difficultés rencontrées dans la manipulation des embryons de moins de $500 \mu \mathrm{m}$ du fait de leur forte teneur en eau, nous avons expérimenté des techniques de culture in vitro de gousses et d'ovules fécondés. De nombreux travaux jouant à la fois sur le «mode» cultural et sur les facteurs du milieu ont permis de cultiver avec succès gousses et ovules fécondés. Ainsi, en ce qui concerne les légumineuses, il est possible de cultiver, en milieu liquide, des gousses et des ovules fécondés de Pisum (SRIVASTAVA et al., 1980), des ovules fécondés dans le genre Glycine (NEwEll \& HyMowitz, 1982). Pour les genres Lens (COHEN et al., 1984), Medicago (MCCOY, 1985) et Lupinus (SATOR, 1985), des cultures d'ovules fécondés, sur milieu solide, ont permis le «sauvetage d'embryons immatures ».

La manipulation des embryons de $1 \mathrm{~mm}$ environ pose peu de problèmes car ceux-ci sont bien visibles et leur teneur en eau plus faible. Il est donc possible de cultiver in vitro de tels embryons sous forme d'embryons isolés. Nous avons cherché à optimiser leur milieu de germination.

\section{MATÉRIEL ET MÉTHODES}

\section{A. Matériel}

Pour L. mutabilis, le génotype étudié est en cours de sélection. Il est noté «LM 34 » et a été sélectionné par J. BERTHAUT dans la collection de la Station d'Amélioration des Plantes de Dijon (Côte-d'Or).

Pour L. albus, les génotypes correspondent à la variété «Lublanc», aux variétés en cours d'études «Ultra », «Lucrop », « Lutop », « Blanca » et «L8 »; tous ces génotypes ont été sélectionnés par M. LENOBLE à la Station d'Amélioration des Plantes fourragères de Lusignan (Vienne).
Le matériel L. mutabilis et L. albus était installé soit en serre soit au champ.

Les croisements en serre ont été réalisés d'avril à juin, les croisements au champ en juillet.

\section{B. Méthodes}

La désinfection des gousses récoltées dans les champs ou dans les serres est faite avec de l'alcool à $95^{\circ}$, sous hotte à flux laminaire.

Dans tous les cas les cultures in vitro se font à l'obscurité, à une température de $20^{\circ} \mathrm{C}$. Le passage à la lumière est progressif ; il débute lorsque la plantule ayant germé atteint 1 à $3 \mathrm{~cm}$.

\section{Culture de gousses}

Des gousses sont prélevées 1 à 4 semaines après déclenchement des fleurs; elles mesurent de 1 à $5 \mathrm{~cm}$. Une fois stérilisées, elles sont fendues sur toute leur longueur et ouvertes. Le ou les côtés portant des ovules sont mis en culture en boîte de Petri de $5 \mathrm{~cm}$ de diamètre, dans $2 \mathrm{ml}$ de milieu liquide. Les gousses ne baignent donc pas complètement dans le milieu. Les boîtes de Petri sont déposées sur un agitateur lent.

\section{Culture d'ovules fécondés}

Nous avons pu constater que, selon les genres et les auteurs, le « mode » cultural est très variable.

En milieu liquide nous avons procédé de 2 manières différentes :

- ovules baignant dans le liquide,

- ovules déposés sur un pont de papier filtre ne trempant pas totalement dans le milieu (technique décrite pour le genre Glycine, en 1982, par NEWELL et HYMOWITZ).

Sur milieu solide nous avons cultivé soit :

- des ovules entiers, déposés à plat sur le milieu ou plantés du côté du hile dans le milieu,

- des demi-ovules, posés sur le milieu ou inclus dans le milieu.

Un demi-ovule est obtenu par coupure de l'ovule fécondé selon la ligne de suture des téguments, il comprend l'albumen et l'embryon.

\section{Culture d'embryons isolés}

Les embryons prélevés aseptiquement sous loupe binoculaire ou non, sont posés sur milieu solide en boîtes de Petri, à raison de plusieurs embryons par boîte.

\section{Milieu (tabl. 1 et 2)}

Le milieu de base utilisé par SATOR (1985) pour la culture in vitro de L. polyphyllus L. et accessoirement de $L$. albus, est le milieu MURASHIGE \& SKOOG (1962). Nous avons aussi testé les milieux de base préconisés pour le sauvetage d'embryons immatures des genres Lens (COHEN et al., 1984) et Trifolium (WILLIAMS \& DE LAUTOUR, 1980). Ces milieux sont nommés par leurs auteurs 100, 110, d'une part, et EC1, d'autre part (tabl. 1).

La nécessité de mettre des hormones dans les milieux ou non, le choix de celles-ci et leurs concen- 
TABLEAU 1

Composition des différents milieux de base.

Chemical composition of media.

\begin{tabular}{|c|c|c|c|}
\hline Code milieux & $\begin{array}{c}169 \text { à } 177 \\
\text { (MuraSHiGe \& SKOOG) }\end{array}$ & 100 et 110 & $\mathrm{EC} 1$ \\
\hline \multicolumn{4}{|l|}{ Macro-éléments (en g/l) } \\
\hline $\begin{array}{l}\mathrm{KCL} \\
\mathrm{NH}_{4} \mathrm{NO}_{3} \\
\mathrm{KNO}_{3} \\
\mathrm{CaCl}_{2} \\
\mathrm{CaNO}_{3} \\
\mathrm{MgSO}_{4}, 7 \mathrm{H}_{2} \mathrm{O} \\
\mathrm{KH}_{2} \mathrm{PO}_{4} \\
\mathrm{Na}_{2} \mathrm{SO}_{4} \\
\mathrm{NaH}_{2} \mathrm{PO}_{4}, 2 \mathrm{H}_{2} \mathrm{O}\end{array}$ & $\begin{array}{l}- \\
1,65 \\
1,90 \\
0,44 \\
- \\
0,37 \\
0,17 \\
- \\
-\end{array}$ & $\begin{array}{l}- \\
1,65 \\
1,90 \\
0,44 \\
- \\
0,37 \\
0,17 \\
-\end{array}$ & $\begin{array}{l}0,065 \\
- \\
0,080 \\
- \\
0,200 \\
0,720 \\
- \\
0,200 \\
0,021\end{array}$ \\
\hline \multicolumn{4}{|l|}{ Micro-éléments (en mg/l) } \\
\hline $\begin{array}{l}\mathrm{H}_{3} \mathrm{BO}_{3} \\
\mathrm{MnSO}_{4}, 1 \mathrm{H}_{2} \mathrm{O} \\
\mathrm{ZnSO}_{4}, 1 \mathrm{H}_{2} \mathrm{O} \\
\mathrm{KI} \\
\mathrm{Na}_{2} \mathrm{MoO}_{4}, 2 \mathrm{H}_{2} \mathrm{O} \\
\mathrm{CuSO}_{4}, 5 \mathrm{H}_{2} \mathrm{O} \\
\mathrm{CoCl}_{2}, 6 \mathrm{H}_{2} \mathrm{O}\end{array}$ & $\begin{array}{c}6,2 \\
13,6 \\
6,6 \\
0,83 \\
0,25 \\
0,025 \\
0,025\end{array}$ & $\begin{array}{c}6,2 \\
13,6 \\
6,6 \\
0,83 \\
0,25 \\
0,025 \\
0,025\end{array}$ & $\begin{array}{l}1,5 \\
5,3 \\
1,9 \\
0,83 \\
0,25 \\
0,025 \\
0,025\end{array}$ \\
\hline \multicolumn{4}{|l|}{ Vitamines (en mg/l) } \\
\hline $\begin{array}{l}\text { Biotine } \\
\text { Thiamine } \mathrm{HCl} \\
\text { Pyridoxine } \mathrm{HCl} \\
\text { Acide nicotinique } \\
\text { Pantothenate de Ca } \\
\text { Chlorhydrate de cystéine }\end{array}$ & $\begin{array}{l}0,01 \\
1 \\
1 \\
1 \\
1 \\
1\end{array}$ & $\begin{array}{l}- \\
0,1 \\
0,3 \\
0,5 \\
- \\
-\end{array}$ & $\begin{array}{l}- \\
0,4 \\
1 \\
5 \\
0,1 \\
-\end{array}$ \\
\hline \multicolumn{4}{|l|}{ Sucres $(\mathrm{en} \mathrm{mg} / \mathrm{l}$ ) } \\
\hline $\begin{array}{l}\text { Myoinositol } \\
\text { Saccharose }\end{array}$ & $\begin{array}{r}100 \\
30000\end{array}$ & $\begin{array}{r}100 \\
30000\end{array}$ & $\begin{array}{r}100 \\
20000\end{array}$ \\
\hline \multicolumn{4}{|l|}{ Fer (en mg/l) } \\
\hline $\begin{array}{l}\mathrm{FeSO}_{4}, 7 \mathrm{H}_{2} \mathrm{O} \\
\mathrm{Na}_{2} \text { EDTA }\end{array}$ & $\begin{array}{l}55,7 \\
74,5\end{array}$ & $\begin{array}{l}37,35 \\
27,85\end{array}$ & - \\
\hline Agar $(e n g / 1)^{(a)}$ & ${ }_{7}(\mathrm{~b})$ & 9 & 7 \\
\hline
\end{tabular}

(a) Difco agar.

(b) quelquefois $5 \mathrm{~g} / 1$ pour la culture de demi-ovule (voir texte) ; sometimes $5 \mathrm{~g} / 1$ for half ovule culture (see text).

TABLEAU 2

Milieux testés.

Media tested.

\begin{tabular}{|c|c|c|c|c|c|c|c|c|c|c|c|c|c|}
\hline Hormones et leur & concentration & 169 & 174 & 170 & 175 & 172 & 176 & 173 & 177 & 100 & 110 & 171 & $\mathrm{ECl}$ \\
\hline ANA & $2 \mathrm{mg} / 1$ & - & - & $x$ & $\times$ & - & - & $x$ & $x$ & - & - & - & - \\
\hline \multirow{2}{*}{ AIA } & $0,2 \mathrm{mg} / 1$ & - & - & - & - & - & - & - & - & $x$ & $x$ & - & - \\
\hline & $0,1 \mathrm{mg} / 1$ & - & - & - & - & - & - & - & - & - & - & $x$ & - \\
\hline BAP & $1,5 \mathrm{mg} / 1$ & - & - & - & - & $\times$ & $\times$ & $x$ & $\times$ & - & - & - & - \\
\hline \multirow[t]{2}{*}{ Zea. } & $0,2 \mathrm{mg} / 1$ & - & - & - & - & - & - & - & - & - & $x$ & - & - \\
\hline & $0,5 \mathrm{mg} / 1$ & - & - & - & - & - & - & - & - & $x$ & - & - & - \\
\hline \multirow{2}{*}{$\begin{array}{l}\text { Cas. } \\
\text { GLN }\end{array}$} & $2 \mathrm{~g} / 1$ & - & $x$ & - & $x$ & - & $x$ & - & $x$ & - & - & - & - \\
\hline & $200 \mathrm{mg} / \mathrm{l}$ & $x$ & $\times$ & $x$ & $x$ & $x$ & $\times$ & $\times$ & $\times$ & - & - & $x$ & - \\
\hline
\end{tabular}

Zea. = Zéatine. Cas. = Hydrolysat de caséine. GLN = Glutamine.

trations sont l'objet essentiel des études sur les milieux de culture in vitro. Les travaux sur L. polyphyllus, Lens et Trifolium ont largement contribué aux choix et aux dosages des hormones que nous avons testées (tabl. 2).
Par ailleurs l'importance de l'hydrolysat de caséine est soulignée par SATOR pour la culture de $L$. polyphyllus. Nous avons nous-mêmes noté un effet très positif de la présence de glutamine dans les milieux de bouturage de L. mutabilis. Nous avons donc testé 
l'effet de ces 2 substances aminées sur le sauvetage d'embryons immatures de lupin (tabl. 2).

Enfin, l'observation des cultures de demi-ovules nous a amenées à modifier la teneur en agar des milieux.

\section{RÉSULTATS}

\section{A. Culture de gousses}

Une vingtaine de gousses de «LM 34 » et de «Lublanc » ont été placées dans des boîtes de Petri contenant le milieu 169. Après 3 à 4 semaines de culture nous observons un très léger grossissement des ovules (par imbibition de la paroi ?). En ouvrant ces ovules nous constatons que l'intérieur est déshydraté. Dans les ovules les plus gros (4 à $5 \mathrm{~mm}$ ), les embryons apparaissent comme de petits amas cellulaires de $500 \mu \mathrm{m}$ environ jaunissants et desséchés.

\section{B. Culture d'ovules entiers (tabl. 3, 4, 5)}

Sur milieux solides, pour les 2 espèces étudiées, quelle que soit la taille des ovules fécondés mis en cul-
TABLEAU 3

Culture d'ovules sur milieu solide.

Nombre d'embryons viables obtenus.

Ovule culture on solid medium. Number of viable embryos.

L. mutabilis (LM 34) L. albus (Lublanc)

\begin{tabular}{|c|c|c|c|c|c|}
\hline $\begin{array}{l}\text { Taille } \\
\text { ovule }\end{array}$ & Milieu & 100 & $\mathrm{EC} 1$ & 169 & 171 \\
\hline $2-3 \mathrm{~mm}$ & & $0 / 24^{(1)}$ & $0 / 20^{(1)}$ & $10 / 10^{(3)}$ & \\
\hline $3-4 \mathrm{~mm}$ & & & & $0 / 24^{(2)}$ & \\
\hline $4-5 \mathrm{~mm}$ & & $0 / 30^{(1)}$ & $0 / 15^{(1)}$ & $0 / 16^{(2)}$ & $0 / 6^{(1)}$ \\
\hline
\end{tabular}

(1) Les ovules sont desséchés et les embryons sont morts à l'intérieur ; ovules dry up and embryos die.

(2) Les ovules ne se dessèchent pas mais ne croissent pas, les embryons jaunissent et ne peuvent être sauvés ; ovules do not dry up but fail to grow ; embryos turn yellow and cannot be rescued.

(3) Contrairement aux autres ovules ceux-ci ont été repris après une semaine de culture et ouverts. Les demi-ovules sont cultivés sur milieu solide ; les embryons ont pu ainsi se développer et germer ; ovules taken up after one week and opened ; half ovules then cultivated on solid medium, so the embryos were able to develop and to germinate.

TABLEAU 4

Culture d'ovules entiers de L. albus ("Lublanc") en milieu liquide. Nombre d'embryons viables obtenus.

L. albus ('Lublanc') ovule culture in liquid medium. Number of viable embryos.

\begin{tabular}{|c|c|c|c|c|c|c|c|c|c|c|}
\hline \multirow[t]{2}{*}{ Milieu } & \multicolumn{2}{|c|}{169} & \multicolumn{2}{|c|}{170} & \multicolumn{2}{|c|}{172} & \multicolumn{2}{|c|}{173} & \multicolumn{2}{|c|}{174} \\
\hline & $\mathbf{P}$ & B & $\mathbf{P}$ & $\mathrm{B}$ & $P$ & B & $P$ & $\mathrm{~B}$ & $P$ & B \\
\hline \multicolumn{11}{|c|}{$\begin{array}{l}\text { Taille des ovules } \\
\text { prélevés }\end{array}$} \\
\hline $2-3 \mathrm{~mm}$ & $0 / 5$ & $0 / 20$ & $0 / 5$ & $0 / 20$ & $0 / 5$ & $0 / 20$ & $0 / 5$ & $0 / 20$ & $0 / 5$ & $0 / 20$ \\
\hline $4-5 \mathrm{~mm}$ & $2 / 5$ & - & $3 / 5$ & - & $2 / 5$ & - & $3 / 5$ & - & $1 / 5$ & - \\
\hline
\end{tabular}

$\mathrm{P}$ : pont ; B : boîte de Petri.

$P$ : bridge ; $B$ : Petri dish.

TABLEAU 5

Cultures d'ovules de L. albus ("Lublanc ") en milieu liquide. Croissance des ovules et taille des embryons après 3 semaines de culture sur pont de papier (croissance mesurée en $\mathrm{mm}$ ).

L. albus ('Lublanc') ovule culture in liquid medium. Ovule and embryo size after 3 weeks of culture on paper bridge (measured in $\mathrm{mm}$ ).

\begin{tabular}{|c|c|c|c|c|c|c|c|c|c|c|}
\hline \multirow[t]{2}{*}{ Milieu } & \multicolumn{2}{|c|}{169} & \multicolumn{2}{|c|}{$\begin{array}{l}169 \mathrm{~A} \\
\text { ANA }\end{array}$} & \multicolumn{2}{|c|}{$\begin{array}{l}\text { 169B } \\
\text { BAP }\end{array}$} & \multicolumn{2}{|c|}{$\begin{array}{c}169 \mathrm{C} \\
\text { ANA + BAP }\end{array}$} & \multicolumn{2}{|c|}{$\begin{array}{l}174 \\
\text { Cas }\end{array}$} \\
\hline & $\mathrm{O}$ & $\mathrm{E}$ & $\mathrm{O}$ & $\mathrm{E}$ & $\mathrm{O}$ & $\mathrm{E}$ & $\mathrm{O}$ & $\mathrm{E}$ & $\mathrm{O}$ & $\mathrm{E}$ \\
\hline \multicolumn{11}{|c|}{ Taille des ovules } \\
\hline \multirow{2}{*}{ prélevés } & 8 & - & 7 & - & 6 & 1 & 8 & 2,5 & 8 & - \\
\hline & 9 & - & 8 & 2 & 8 & - & 9 & 2 & 8 & - \\
\hline \multirow[t]{5}{*}{$4-5 \mathrm{~mm}$} & 7 & 0,8 & 8 & 2 & 8 & 2 & 9 & 5 & 8 & - \\
\hline & 9 & - & 9 & 1 & 10 & - & 10 & - & 7 & 1 \\
\hline & 9 & 2 & 9 & - & 10 & - & 9 & - & 8 & - \\
\hline & 4 & - & 6 & - & 4 & - & 4 & - & 4 & - \\
\hline & 5 & - & 4 & - & 4 & - & 4 & - & 4 & - \\
\hline \multirow[t]{3}{*}{$2-3 \mathrm{~mm}$} & 4 & - & 4 & - & 4 & - & 4 & - & 4 & - \\
\hline & 5 & - & 4 & - & 4 & - & 4 & - & 4 & - \\
\hline & 5 & - & 4 & - & 4 & - & 4 & - & 4 & - \\
\hline
\end{tabular}

0 : taille des ovules ; ovules size. E : taille des embryons, après 3 semaines de culture ; embryo size, after 3 weeks of culture.

- : embryon desséché, dried up embryo. 
ture ou la position des ovules sur le milieu, nous n'avons observé aucune croissance (tabl. 3).

En milieu liquide nous n'avons étudié que $L$. albus. La croissance embryonnaire dépend non seulement de la taille des ovules mais aussi de l'absence de contact direct avec le milieu (méthode dite "du pont ", tabl. 4). En effet quand les ovules baignent dans le milieu liquide, il y a un léger gonflement des ovules et dessèchement des embryons, comme cela a été observé pour la culture de gousse en milieu liquide.

Lorsque les ovules fécondés sont cultivés sur pont de papier filtre, l'analyse de la taille des ovules et des embryons après 3 semaines de culture en milieu liquide (tabl. 5) montre :

- que les différences de teneurs en hormones et hydrolysat de caséine des milieux n'ont pas d'effet sur la croissance des ovules ;

- que les plus gros embryons sont obtenus avec le milieu contenant de l'A.N.A. et de la B.A.P. (milieu 173), dans le cas d'ovules mesurant de 4 à $5 \mathrm{~mm}$ lors de la mise en culture ; pour les ovules plus petits ( 2 à $3 \mathrm{~mm}$ ), les embryons sont desséchés.

Après 3 semaines, les embryons prélevés et mesurés sont repiqués sur milieu solide (le milieu de repiquage est le milieu de culture des ovules). Pour rendre ces milieux solides, $7 \mathrm{~g} / \mathrm{l}$ d'agar sont ajoutés. Le repiquage sur les milieux contenant des hormones (170, $172,173)$ entraîne une callogenèse des embryons immatures.

\section{Culture de demi-ovules (tabl. 6)}

Cette technique a été utilisée pour $L$. albus exclusivement.

Les demi-ovules sont inclus dans ou posés sur les milieux, que ceux-ci contiennent $7 \mathrm{~g} / \mathrm{l}$ ou $5 \mathrm{~g} / \mathrm{l}$ d'agar.

\section{TABLEAU 6}

Culture de demi-ovules de $\mathrm{L}$. albus inclus dans un milieu solide. Nombre d'embryons viables/nombre de demi-ovules.

L. albus half ovule culture in solid medium. Number of viable embryos/number of half ovules.

\begin{tabular}{|c|c|c|}
\hline Milieu & $\begin{array}{c}169 \\
+ \\
5 \mathrm{~g} / 1 \mathrm{~d} \text { 'agar }\end{array}$ & $\begin{array}{c}169 \\
+ \\
7 \mathrm{~g} / 1 \text { d'agar }\end{array}$ \\
\hline $\begin{array}{c}\text { Taille des ovules prélevés } \\
\text { Génotype }\end{array}$ & $3-4 \mathrm{~mm}$ & $4-5 \mathrm{~mm}$ \\
\hline L 8 & - & $0 / 6$ \\
\hline Ultra & $9 / 12$ & $0 / 6$ \\
\hline Lublanc & $10 / 16$ & $0 / 6$ \\
\hline Lucrop & - & $0 / 6$ \\
\hline Blanca & $9 / 9$ & $0 / 6$ \\
\hline Lutop & $8 / 12$ & $0 / 6$ \\
\hline
\end{tabular}

Sur le milieu 169 avec 5 g/l d'agar, 9 à 16 demiovules sont mis en culture pour chacun des génotypes. La désinfection des génotypes L8 et «Lucrop », prélevés comme les autres au champ, n'a pas été réussie malgré 2 tentatives qui portent l'échantillonnage pour ces 2 génotypes à 24 demi-ovules.

Sur le milieu 169 avec $7 \mathrm{~g} / \mathrm{l}$ d'agar, la désinfection a posé beaucoup moins de problèmes.
Dans les demi-ovules, lors de la mise en culture, les embryons sont au stade cordiforme et mesurent de 200 à $500 \mu \mathrm{m}$. Ils atteignent 2 à $3 \mathrm{~mm}$ après 3 semaines de culture et sont alors repiqués en tube.

Seule l'inclusion dans le milieu à faible teneur en agar (169 avec $5 \mathrm{~g} / \mathrm{l}$ d'agar) permet le développement des embryons. L'inclusion dans le milieu à $7 \mathrm{~g} / \mathrm{l}$ agar ou le dépôt sur les milieux provoquent la déshydratation des embryons.

Par ailleurs, cette expérience ne fait apparaître aucun effet génotypique.

\section{Culture d'embryons (tabl. 7)}

L. mutabilis a été la première des 2 espèces à être étudiée. Tenant compte des travaux de SATOR (1985), de Williams \& DE LAUTOUR (1980) et de COHEN et al. (1984), nous avons choisi les milieux 169, 174, 175, $176,177, \mathrm{EC} 1$ et 110 pour tester l'effet des hormones sur le sauvetage d'embryons immatures.

Les milieux qui permettent le développement des embryons immatures de $L$. mutabilis sont les milieux

TABLEAU 7

Germination des embryons. Embryo germination.

\begin{tabular}{|c|c|c|c|c|}
\hline \multirow[b]{2}{*}{ Génotype } & \multicolumn{2}{|c|}{ Embryon de 0,5 à $1 \mathrm{~mm}$} & \multicolumn{2}{|c|}{ Embryon de 1 à $2 \mathrm{~mm}$} \\
\hline & LM 34 & Lublanc & LM 34 & Lublane \\
\hline \multicolumn{5}{|l|}{ Milieu } \\
\hline 169 & $38 / 46$ & $3 / 15+4 / 6(1)$ & $16 / 22$ & $6 / 18+6 / 8\left(^{1}\right)$ \\
\hline 174 & $3 / 10$ & $0 / 10$ & $2 / 5$ & $0 / 4$ \\
\hline $\mathrm{ECl}$ & $0 / 10$ & - & $4 / 10$ & \\
\hline $169 \mathrm{~A}$ & - & $0 / 8 \quad$ (cal) & - & $0 / 8$ (cal) \\
\hline 175 & $0 / 10$ (cal) & - & $0 / 5 \quad$ (cal) & - \\
\hline 169B & - & $0 / 12$ (cal) & - & $0 / 6 \quad$ (cal) \\
\hline 176 & $0 / 10$ (cal) & - & $0 / 5$ & - \\
\hline $169 \mathrm{C}$ & - & $0 / 12$ & - & $0 / 12$ \\
\hline 177 & $0 / 10$ (cal) & - & $0 / 5$ & - \\
\hline 110 & $0 / 24$ & - & $0 / 2$ & - \\
\hline
\end{tabular}

\begin{tabular}{|c|c|c|}
\hline \multicolumn{3}{|c|}{ Embryon de 2 à $5 \mathrm{~mm}$} \\
\hline Génotype & LM 34 & Lublanc \\
\hline \multicolumn{3}{|l|}{ Milieu } \\
\hline 169 & $21 / 29$ & $3 / 3+10 / 12(1)$ \\
\hline 174 & $2 / 10$ & $0 / 9$ \\
\hline $\mathrm{EC} 1$ & $7 / 7$ & - \\
\hline $169 \mathrm{~A}$ & - & $0 / 6$ (cal) \\
\hline 175 & $0 / 10$ (cal) & - \\
\hline 169B & - & $0 / 2$ (cal) \\
\hline 176 & $0 / 10(\mathrm{cal})$ & - \\
\hline $169 \mathrm{C}$ & - & $0 / 5$ (cal) \\
\hline 177 & $0 / 10$ (cal) & - \\
\hline 110 & $0 / 8$ & - \\
\hline
\end{tabular}

(1) Ces embryons ont d'abord été déposés sur milieu $169+5 \mathrm{~g}$ d'agar. Au bout d'une semaine ils ont été repiqués sur milieu $169+7 \mathrm{~g}$ d'agar car nous observions leur jaunissement ou leur brunissement.

cal : tous ces cals ne sont pas identiques entre eux et ne ressemblent pas non plus à ceux obtenus à partir de fragment de tige ou à partir de pétiole.

Embryos first placed on medium $169+5 \mathrm{~g}$ of agar ; after one week removed to medium $169+7 \mathrm{~g}$ of agar because they became either yellow or brown.

cal : calluses not identical nor like calluses obtained from stem fragments or from petiole. 
169,174 et EC1. Ces 3 milieux ne contiennent pas d'hormones. Le milieu 169 est le meilleur des 3. Le milieu EC1 est d'autant plus efficace que les embryons mis en culture sont plus gros.

Pour L. albus, nous avons choisi de tester uniquement des milieux dont les macro- et les microéléments sont ceux de Murashige et Skoog. Nous avons pris le parti de ne pas rajouter de l'hydrolysat de caséine aux milieux avec hormones. En conséquence, les milieux testés sont les milieux 169, 174, 170, 172, 173. Comme pour $L$. mutabilis, les milieux avec hormones ne permettent pas le développement normal des embryons. Mais des 2 milieux sans hormones, seul le milieu 169 qui ne contient pas d'hydrolysat de caséine permet de voir les embryons immatures germer.

Cependant nous n'avons pu conduire les germinations de "Lublanc" à leur terme car les plantules obtenues se mettent rapidement à jaunir et meurent. Ce phénomène de jaunissement a été observé sur les microboutures de divers génotypes de $L$. albus que nous avons tenté d'obtenir.

\section{CONCLUSION}

La germination des embryons d'environ $1 \mathrm{~mm}$ et plus est aisée à obtenir, pourvu que les milieux ne contiennent pas d'hormones.

Cependant, dans le cas de $L$. albus, il est difficile d'obtenir la survie des plantules car elles jaunissent. Cela est peut-être dû à la teneur en calcium du milieu, bien que cette espèce soit considérée comme indifférente par LAMANT \& HELLER (1975).
Le sauvetage des embryons plus petits, hors des ovules, n'a pas été étudié ici à cause des difficultés de prélèvement.

La culture d'ovules, qui a été étudiée presque exclusivement chez $L$. albus, est possible pourvu que ces ovules mesurent déjà au moins $3 \mathrm{~mm}$. Ceci correspond à des embryons compris entre 200 et $500 \mu \mathrm{m}$ pour L. albus.

La principale difficulté rencontrée est le dessèchement précoce des embryons puis des ovules. Deux techniques nous ont permis de trouver une solution à ce problème : la culture d'ovules sur pont de papier filtre trempant dans un milieu liquide et la culture de demi-ovules noyés dans un milieu à faible teneur en agar.

Contrairement à la culture d'embryons, les teneurs en hormones des milieux ont peu d'effets sur la croissance des ovules et de leur embryon. Toutefois le repiquage des embryons de 3 à $5 \mathrm{~mm}$ sur les mêmes milieux conduit à la formation de cals ou à des désorganisations très importantes de la morphologie des plantules. Il apparaît done que le milieu le plus simple est le mieux adapté au sauvetage des embryons de lupin.

Les hybridations interspécifiques réalisées montrent que les embryons obtenus meurent le plus souvent bien avant d'avoir atteint le stade de $200 \mu \mathrm{m}$ (VUILLAUME \& HOFF, 1986). Quelques essais s'inspirant de la technique «culture sur albumen nourricier » décrite par WILliaMS \& DE LAUTOUR (1980) pour Trifolium ont été tentés. Pour l'instant nous n'avons pas réussi mais nous ne savons pas si cet échec peut être imputé à la méthode ou aux difficultés de prélèvement des embryons de moins de $200 \mu \mathrm{m}$.

Reçu le 14 octobre 1985. Accepté le 8 août 1986.

\section{RÉFÉRENCES BIBLIOGRAPHIQUES}

Cohen D., Ladizinsky G., Ziv M., Muehlbauer F. J., 1984. Rescue of interspecific Lens hybrids by means of embryo culture. Plant Cell Tissue Organ Culture, 3, 343-347.

Jaranovski J., 1962. Fertilization and embryo development in the genus Lupinus. Part I : Seed development in cases of autogamy. Part II : Fertilization and embryo development following reciprocal species hybridization. Genetica Polonica, 3, 210-246, 333-368.

Lamant A., Heller R., 1975. Intervention des systèmes membranaires dans l'absorption du calcium par les racines de féverole (calcicole) et de lupin (calcifuge). Physiol. Veg., 13, 685-700.

McCoy T. J., 1985. Interspecific hybridization of Medicago sativa L. and $M$. rupestris M. B. using ovule-embryo culture. Can. J. Genet. Cytol., 27, 238-245.

Murashige T., Skoog A., 1962. A revised medium for rapid growth and bioassays with tobacco tissue cultures. Physiol. Plant., 15, 473497.
Newell C. A., Hymowitz T., 1982. Successful wide hybridization between the soybean and a wild perennial relative. $G$. tomentella Hayata. Crop Sci., 22, 1062-1065.

Sator Ch., 1985. Regeneration von Lupinenpflanzen aus Embryonen. Landbauforsch. Völkenrode, 35, 1-4.

Srivastava P. S., Varga A., Bruinsma J., 1980. Growth in vitro of fertilized ovules of pea, Pisum sativum L. with and without pods. Z. Pflanzenphysiol., Bd 98 S, 347-354.

Vuillaume E., Hoff T., 1986. Essais d'hybridation interspécifique entre Lupinus albus L. et Lupinus mutabilis Sweet. Influence des conditions de culture et du génotype. Agronomie, 6 (10), 919-924.

Williams E. G., De Lautour G., 1980. The use of embryo culture with transplanted nurse endosperm for the production of interspecific hybrids in pasture legumes. Bot. Gaz., 141, 252-257.

Williams W., Akhtar M. A., Faluyi M., 1980. Cross compatibility between European and American Lupinus species. Bot. J. Linn. Soc., $81,225-232$. 